\title{
Angiotensin II evokes hypotension and renal sympathoinhibition from a highly restricted region in the nucleus tractus solitarii
}

\author{
Peter S.P. Tan, Jason R. Potas, Suzanne Killinger, Jouji Horiuchi, Ann K. Goodchild, \\ Paul M. Pilowsky, Roger A.L. Dampney*
}

Department of Physiology and Institute for Biomedical Research, The University of Sydney, Sydney, NSW 2006, Australia

Accepted 4 December 2004

\begin{abstract}
Microinjections of low doses (in the femtomolar or low picomolar range) of angiotensin II (Ang II) into the nucleus tractus solitarii (NTS) evoke depressor responses. In this study we have mapped in the rat the precise location of the subregion within the NTS at which Ang II evokes significant sympathoinhibitory and depressor responses. Microinjections of 1 pmol of Ang II evoked large decreases ( $\geq 20 \%$ of baseline) in renal sympathetic nerve activity (RSNA), from a highly restricted region in the medial NTS, at or very close to the level $0.2 \mathrm{~mm}$ caudal to the obex. Microinjections of the same dose of Ang II into the commissural or lateral NTS at the same rostrocaudal level, or into the medial and lateral NTS at the level of the obex evoked significantly smaller sympathoinhibitory responses, while microinjections into more rostral or caudal levels of the NTS evoked significant sympathoinhibitory responses even less frequently. In most cases (71\%), the sympathoinhibitory responses were accompanied by depressor responses, the magnitudes of which were also greater within the medial NTS at the level $0.2 \mathrm{~mm}$ caudal to obex, as compared to the surrounding subregions. The results demonstrate that the cardiovascular effects of Ang II in the NTS are highly site-specific. Taken together with previous studies, the results also indicate that the neurons in the NTS that mediate the Ang II-evoked sympathoinhibition are a discrete subgroup of the population of sympathoinhibitory neurons within the nucleus.
\end{abstract}

(C) 2005 Elsevier B.V. All rights reserved.

Theme: Endocrine and autonomic regulation

Topic: Cardiovascular regulation

Keywords: Angiotensin II; Angiotensin type 1 receptor; Nucleus tractus solitarii; Renal sympathetic nerve activity; Cardiovascular control

\section{Introduction}

It is well known that angiotensin II (Ang II) can act on specific receptors within the nucleus tractus solitarii (NTS) to produce changes in blood pressure and heart rate, and also inhibit the baroreceptor reflex control of heart rate (for review see [6]). Microinjections of low doses (in the femtomolar or low picomolar range) of Ang II into the NTS result in a decrease in arterial blood pressure and heart rate $[5,22]$. One study has also shown that the depressor

\footnotetext{
* Corresponding author. Fax: +61 293516470.

E-mail address: rogerd@physiol.usyd.edu.au (R.A.L. Dampney).
}

response to Ang II microinjection is accompanied by a decrease in renal sympathetic nerve activity (RSNA) [16]. The depressor effects of Ang II are mediated by Ang II type 1 (AT1) receptors in the NTS $[7,13]$. There is a high density of AT1 receptors in the NTS $[23,27]$, which are located on both neuronal cell bodies and vagal afferent fibers within the nucleus $[6,9,11,14,28]$. Activation of both presynaptic and postsynaptic AT1 receptors appears to contribute to the hypotensive effects of exogenous Ang II in the NTS [6].

The density of AT1 receptors, as determined using autoradiography, is not uniform within the NTS, varying according to the rostrocaudal level $[1,10]$. Similarly, Sangaleti et al. [24] found that mRNA expression for the AT1A receptor subtype within the NTS also varied 
markedly according to the rostrocaudal level. These observations therefore raise the question as to whether there are also variations in the cardiovascular effects of Ang II within different subregions of the NTS, and in particular whether the sympathoinhibitory actions of Ang II are mediated by neurons in a specific subregion or subregions within the nucleus. For this purpose, we have systematically mapped the effects on mean arterial pressure (MAP), RSNA, and heart rate (HR) of microinjections of a low dose $(1 \mathrm{pmol})$ of Ang II into different subnuclei at different rostrocaudal levels within the NTS and adjacent regions.

\section{Materials and methods}

All experiments were carried out on male SpragueDawley rats (380-550 g, Laboratory Animal Services, University of Sydney, NSW, Australia). All experimental procedures were approved by the Animal Ethics Committee of the University of Sydney. Rats were anesthetized with urethane $(1.4 \mathrm{~g} / \mathrm{kg}$, ip, with supplementary doses of $0.1-0.2 \mathrm{~g} / \mathrm{kg}$ i.v. if required). Body temperature was maintained in the range of $36.5-37.5{ }^{\circ} \mathrm{C}$ with a heating pad. The trachea was cannulated and catheters were placed in a femoral artery and a femoral vein. The MAP and HR were derived from the pulsatile arterial pressure signal by means of a low pass filter and rate meter, respectively. The renal sympathetic nerve on the left side was isolated from surrounding connective tissues and its activity was recorded by a bipolar stainless-steel electrode as described previously [20]. The signal from the electrodes was amplified, passed through a band pass filter $(100-1000 \mathrm{~Hz})$, and then rectified and integrated (resetting every $5 \mathrm{~s}$ ). The baseline noise level of RSNA was determined at the end of the experiment by applying lignocaine $(2 \% \mathrm{w} / \mathrm{v})$ to the proximal end of the renal nerve on the recording electrodes or by intravenous infusion of $0.5 \mathrm{ml}$ of $5 \%$ hexamethonium (Sigma-Aldrich Company).

After completion of all surgical procedures, neuromuscular blockade was induced with alcuronium chloride $(0.2 \mathrm{mg} / \mathrm{kg}$ i.v. every $1-2 \mathrm{~h})$ and the animals were artificially ventilated at a level that maintained end-tidal $\mathrm{CO}_{2}$ close to $4 \%$. The effects of alcuronium chloride were allowed to wear off before each additional dose was administered. The adequacy of anesthesia without neuromuscular blockade was verified by the absence of a withdrawal response to nociceptive stimulation of a hind paw and during neuromuscular blockade by a stable baseline arterial pressure, HR, and RSNA. The MAP, HR, and RSNA were recorded continuously on a computer (PowerLab system, AD Instruments).

The rat was fixed in a stereotaxic apparatus with the head flexed forward at an angle of $45^{\circ}$. The dorsal surface of the medulla was exposed and microinjections of Ang II solution (50 or $1000 \mathrm{fmol}$ in $50 \mathrm{nl}$ ) were made into sites within the medulla using a single-barreled micropipette held in a micromanipulator at an angle of $13^{\circ}$ (tip rostral). The vehicle solution was artificial cerebrospinal fluid (aCSF) adjusted to $\mathrm{pH}$ 7.4. Injections were made by pressure, and the volume injected was measured by the displacement of the meniscus in the pipette with respect to a horizontal grid viewed through an operating microscope. The microinjection was made over a period of approximately $30 \mathrm{~s}$. In all but 5 experiments, a fluorescent marker was added to the injectate for later identification of the injection sites. In the remaining experiments, a double-barreled micropipette was used, and the Ang II injection sites were marked by injection of $0.3 \%$ Evans blue from the second barrel of the micropipette. The calamus scriptorius was used as the rostrocaudal reference point for microinjections. For later mapping of the injection sites, however, the obex was used as the rostrocaudal reference point, as this could be identified more readily in the histological sections.

In each individual experiment, microinjections into the NTS were usually made in one mediolateral plane (ranging from the midline to $1.8 \mathrm{~mm}$ lateral), and were spaced at least $0.4 \mathrm{~mm}$ apart in the rostrocaudal plane, and $0.25 \mathrm{~mm}$ apart in the dorsoventral plane. The centers of the injection sites extended from the level $1.1 \mathrm{~mm}$ rostral to the obex to 1.2 $\mathrm{mm}$ caudal to the obex, and covered the mediolateral and dorsoventral extent of the NTS within this rostrocaudal extent. There was a waiting period of at least $10 \mathrm{~min}$ between microinjections when no response was evoked, and of at least $30 \mathrm{~min}$ if a response was evoked, to allow for the MAP, HR, and RSNA to return to and stabilize at their resting levels.

At the end of the experiment, the rat was killed with an overdose of pentobarbital sodium. The brain was removed and placed in $4 \%$ paraformaldehyde in $0.1 \mathrm{M}$ phosphatebuffered saline (PBS, pH7.4) at $4{ }^{\circ} \mathrm{C}$ for at least $24 \mathrm{~h}$. Following this period of fixation, the brain was placed in $30 \%$ sucrose in PBS for another $24 \mathrm{~h}$ at $4{ }^{\circ} \mathrm{C}$. Two series of $48 \mu \mathrm{m}$-thick coronal sections of brainstem tissue were cut on a carbon dioxide freezing microtome. One series was used to view the fluorescent-labeled injection sites while the second series was stained with neutral red to identify the boundaries of the subnuclei of the NTS and of the surrounding nuclei. The commissural NTS was defined according to the atlas of Paxinos and Watson [19]. The medial NTS was defined as the region of the NTS lateral to the commissural NTS, but medial to the solitary tract, while the lateral NTS was defined as the region lateral to the solitary tract. The centers of the injection sites were mapped on to standard coronal sections of the NTS, which ranged from $1.0 \mathrm{~mm}$ caudal to $1.0 \mathrm{~mm}$ rostral to the obex.

The baseline pre-injection values for MAP, HR, and RSNA were measured as the average values for each of these variables over the $1 \mathrm{~min}$ period immediately preceding the microinjection of Ang II into each site. Comparisons of the changes in MAP, HR, and RSNA evoked by microinjections of Ang II into different subregions were made using ANOVA, while responses evoked by microinjections 
of Ang II into two different regions (or of two different doses) were compared using an unpaired $t$ test, with application of Holm's step-down procedure [26] for multiple comparisons where appropriate. A $P$ value of $<0.05$ was regarded as statistically significant. All values are presented as means $\pm \mathrm{SEM}$.

\section{Results}

Microinjections of Ang II (1pmol) were made into 230 sites in the NTS and surrounding regions in 30 animals. The baseline MAP and HR were $83 \pm 2.6 \mathrm{~mm} \mathrm{Hg}$ and $369 \pm 10$ bpm, respectively. At all sites, Ang II microinjections evoked either no significant change in RSNA $(<10 \%$ of baseline), or else a significant decrease in RSNA ( $\geq 10 \%$ of baseline). An increase in RSNA was not evoked from any site. In most cases (71\%), the sympathoinhibitory responses were accompanied by a decrease in MAP $\geq 10 \mathrm{~mm} \mathrm{Hg}$
(Figs. 1,2), while in the remainder there was no significant change in MAP $(<10 \mathrm{~mm} \mathrm{Hg})$. Similarly, the sympathoinhibitory responses were in most cases $(56 \%)$ accompanied by a bradycardia (e.g. Fig. 1), although in many cases $(35 \%)$ there was little change in HR $(<10 \mathrm{bpm})$ while in the remainder there was a significant increase in HR $(>10$ bpm). Typically, the RSNA began to decrease 10-20 s after the start of injection and the peak response occurred after $45-120 \mathrm{~s}$ (Fig. 1). When the decrease in RSNA was accompanied by a decrease in MAP, the latter showed a similar time course (e.g. Fig. 1A). In contrast, however, the time course of the bradycardia, when it occurred, was often different to that of the sympathoinhibition (e.g. Fig. 1A). The MAP, RSNA, and HR returned to their baseline levels after $10-15 \mathrm{~min}$.

Fig. 2 shows the distribution of injection sites at different rostrocaudal levels ranging from $1 \mathrm{~mm}$ caudal to $1 \mathrm{~mm}$ rostral to the obex, at which responses of different magnitudes were evoked by Ang II microinjections. Large
A
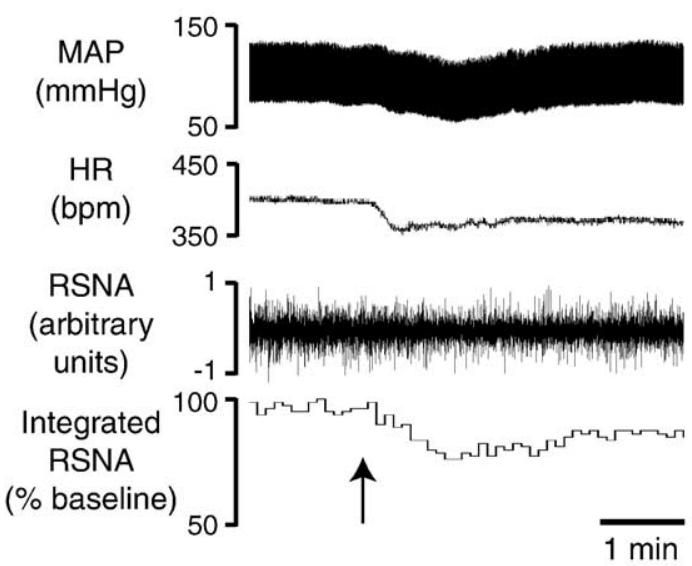

B
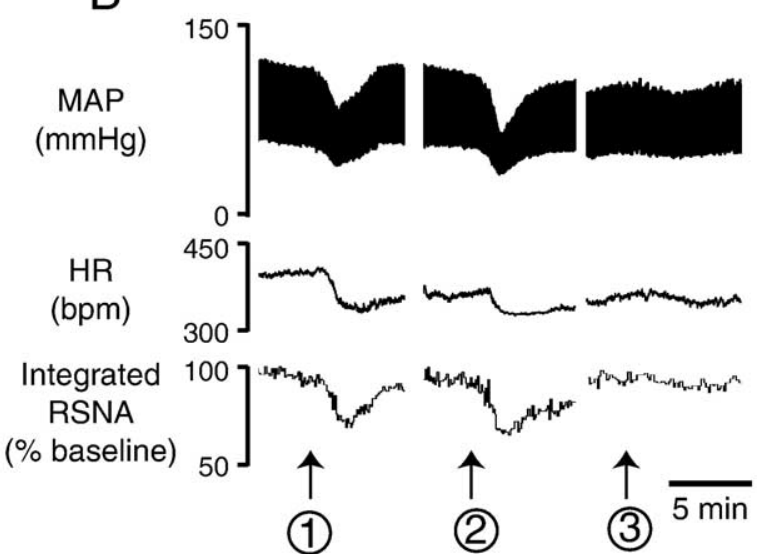
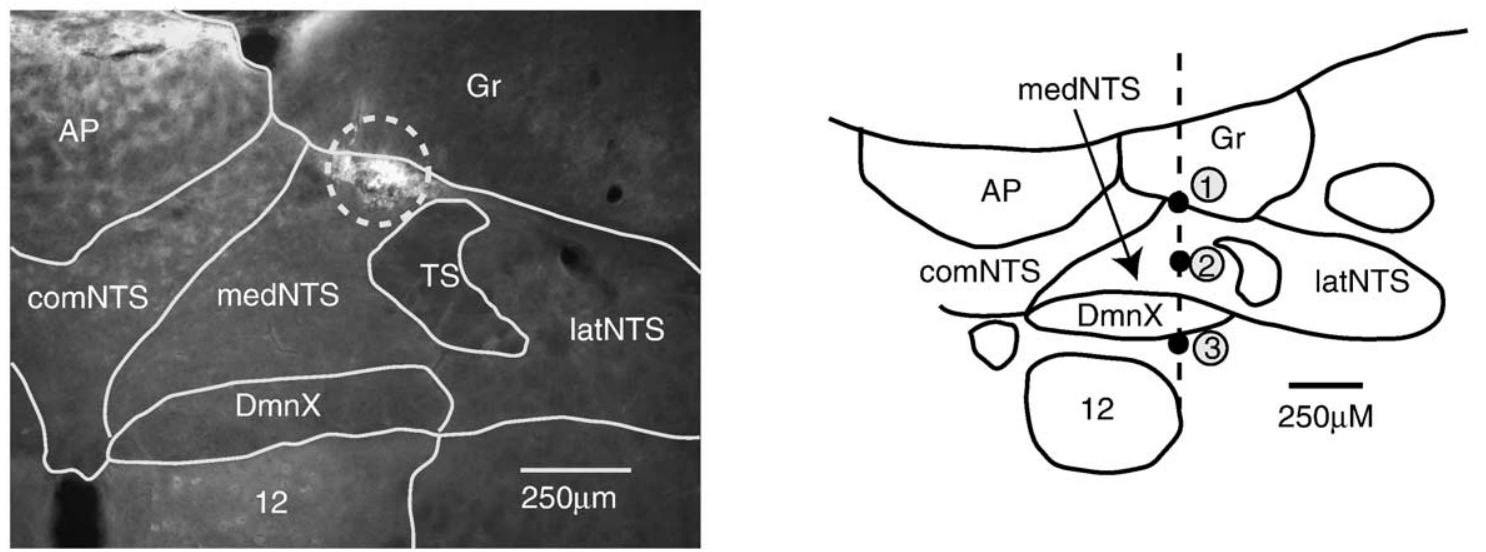

Fig. 1. Examples of responses evoked by microinjection of Ang II (1 pmol) into different sites in the nucleus tractus solitarii (NTS). (A) Changes in arterial pressure (AP), heart rate (HR), and renal sympathetic nerve activity (RSNA) evoked by microinjection of Ang II into the site marked by a fluorescent tracer (indicated by the surrounding circle) in the photomicrograph below. This section was at the level $0.2 \mathrm{~mm}$ caudal to the obex. (B) Responses evoked by microinjection of Ang II (1 pmol) into three sites in a track (dashed line) passing through the NTS at the level 0.2 mm caudal to the obex. The centers of the injection sites are indicated by the numbered filled circles in the drawn outline of the section below. In each case, the arrow marks the time at which the microinjection commenced. Abbreviations: 12; hypoglossal nucleus; AP; area postrema; comNTS; commissural NTS; DmnX; dorsal motor nucleus of the vagus; Gr; gracile nucleus; latNTS, lateral NTS; medNTS; medial NTS; TS; tractus solitarii. 

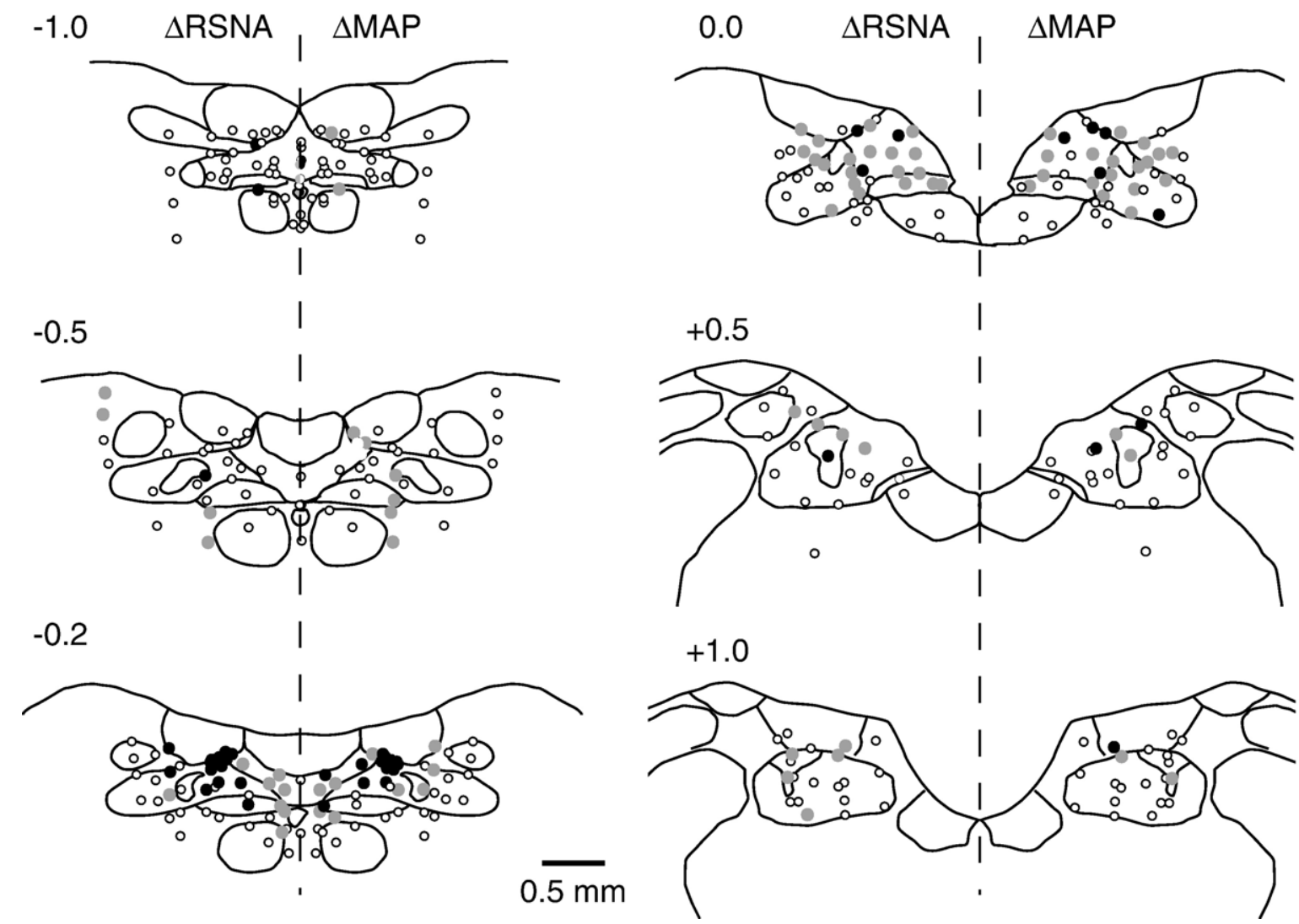

Fig. 2. Series of coronal sections of the dorsomedial medulla showing locations of the centers of injection sites. The levels of the sections (in mm) rostral ( + ) or caudal (-) to the obex are indicated in the upper left corner of each section. The magnitudes of the evoked changes in RSNA (left side) and MAP (right side) evoked at each site are indicated as follows: open circles, change of $<10 \%$ baseline (RSNA) or $10 \mathrm{~mm} \mathrm{Hg}$ (MAP); filled gray circles, decrease of 10-19\% baseline or $10-19 \mathrm{~mm} \mathrm{Hg}$; filled black circles, decrease of $>20 \%$ or $20 \mathrm{~mm} \mathrm{Hg}$.

sympathoinhibitory and depressor responses (decreases in RSNA $\geq 20 \%$ and in MAP of $\geq 20 \mathrm{~mm} \mathrm{Hg}$ ) were most commonly evoked from sites in the medial NTS at the level just caudal to obex $(0.1-0.3 \mathrm{~mm}$ caudal, plotted on to a standard section at $0.2 \mathrm{~mm}$ caudal). Microinjections of Ang II into sites centered only $0.25 \mathrm{~mm}$ apart could evoke responses of markedly different magnitudes. For example, as shown in Fig. 1B, microinjections into three sites $(0.25$ $\mathrm{mm}$ apart) in a track passing through the medial NTS at the level $0.2 \mathrm{~mm}$ caudal to obex evoked large sympathoinhibitory and depressor responses from the two more dorsal sites, whereas Ang II microinjection into the more ventral site, located outside the medial NTS, evoked no significant response.

Moderate sympathoinhibitory and depressor responses (decreases in RSNA of $10-19 \%$ and in MAP of $10-19 \mathrm{~mm}$ $\mathrm{Hg}$ ) were also evoked from nearly all sites in the commissural NTS at the level $0.2 \mathrm{~mm}$ caudal to obex, and from sites in the medial NTS at the level of the obex (Fig. 2). The mean decrease in RSNA evoked from sites in the medial NTS at the level $0.2 \mathrm{~mm}$ caudal to obex was significantly greater than that evoked from sites in the commissural NTS and lateral NTS at the same level, and from the medial and lateral NTS at the level of the obex $(P<0.05$ in all cases) (Fig. 3). Similar differences between subregions were also observed with respect to the magnitude of depressor responses evoked from sites within these different subregions (Fig. 3).

At more rostral and more caudal levels of the NTS $(0.5$ and $1.0 \mathrm{~mm}$ rostral, and 0.5 and $1.0 \mathrm{~mm}$ caudal to obex),

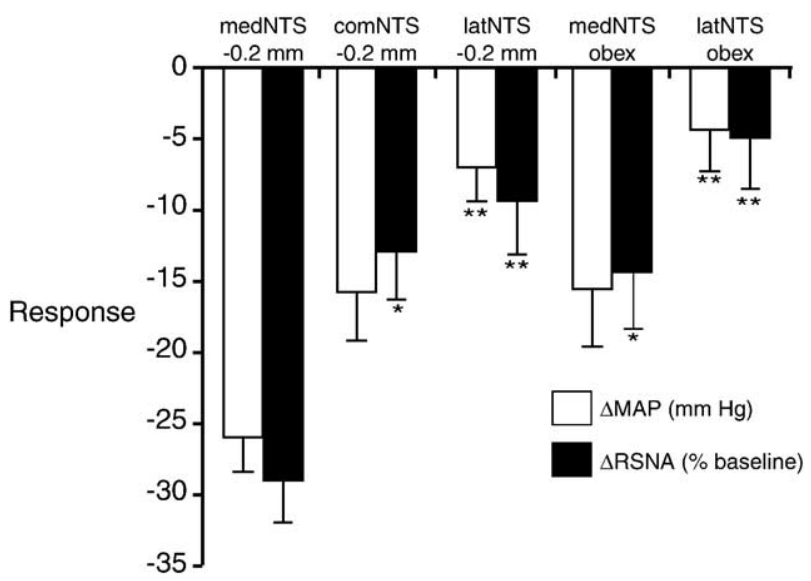

Fig. 3. Histogram showing the magnitude of changes (mean \pm SEM) in MAP and RSNA evoked by microinjection of 1 pmol of Ang II into different subregions of the NTS at the level of the obex and $0.2 \mathrm{~mm}$ caudal to the obex. $* P<0.05$ and $* * P<0.01$, vs. response evoked from the medial NTS at the level $0.2 \mathrm{~mm}$ caudal to obex. 


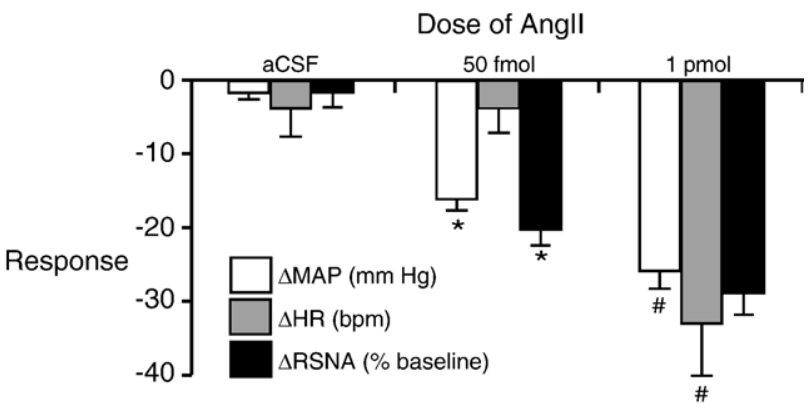

Fig. 4. Histogram showing the magnitude of changes (mean \pm SEM) in MAP, HR, and RSNA evoked by microinjection of two different doses of Ang II or of the vehicle solution (artificial cerebrospinal fluid, aCSF) into the medial NTS at the level $0.2 \mathrm{~mm}$ caudal to the obex. ${ }^{*} P<0.05$ vs. response evoked by aCSF injection; ${ }^{\#} P<0.05$ vs. response evoked by 50 fmol of Ang II.

moderate sympathoinhibitory and depressor responses were evoked much less frequently, while large responses were evoked only occasionally (Fig. 2).

The effects of microinjection of a much smaller dose (50 fmol) of Ang II into the medial NTS at the level 0.2 $\mathrm{mm}$ caudal to obex was also determined. As shown in Fig. 4, 50 fmol of Ang II $(n=6)$ also evoked significant decreases in MAP and RSNA, but not in HR. The magnitudes of the decreases in MAP and RSNA were less than with the higher dose of 1 pmol $(n=13)$, although this difference was not statistically significant in the case of RSNA (Fig. 4). Microinjections of the vehicle aCSF solution $(n=10)$ had no significant effect on MAP, HR, or RSNA (Fig. 4).

\section{Discussion}

This study shows for the first time that microinjections of a low dose of Ang II consistently evokes a marked sympathoinhibition and decrease in arterial pressure from a highly restricted region within the medial part of the NTS, at the level just caudal to the obex. Within this region, even a very low dose of Ang II (50 fmol) was sufficient to evoke significant decreases in both MAP $(\sim 15 \mathrm{~mm} \mathrm{Hg})$ and RSNA $(\sim 20 \%)$. Ang II microinjections within the adjacent commissural subregion of the NTS, at the same rostrocaudal level and at the level of the obex evoked significant but smaller depressor and sympathoinhibitory responses, whereas Ang II microinjections into NTS sites outside this region only occasionally evoked significant decreases in MAP and RSNA.

The effective area of neuronal activation by Ang II following microinjection depends upon a number of factors, including the volume of the injectate (a sphere of volume 50 $\mathrm{nl}$ has a radius of $0.23 \mathrm{~mm}$ ), the density of Ang II receptors within the region, the rate of diffusion of Ang II molecules, and the effectiveness of mechanisms that inactivate Ang II. With regard to the latter, Ang II in the brain is degraded by aminopeptidase A to Ang III with a half-life of approxi- mately 24 s, and Ang III is then further degraded by aminopeptidase B to inactive shorter-length peptides with a half-life of approximately $8 \mathrm{~s}$ [32]. It is difficult to estimate the effects of biochemical degradation as well as other factors that determine the effective area of neuronal activation by Ang II, but the fact that the centers of the Ang II microinjection sites at which significant depressor and sympathoinhibitory responses were evoked were confined to a highly restricted region indicates that, for each microinjection, the response was due primarily to activation of receptors in close proximity to the center of the injection site. In many cases, marked differences in the magnitude of responses were evoked by injection into sites whose centers were only $0.25 \mathrm{~mm}$ apart (e.g. Fig. 1).

The rats were anesthetized by intraperitoneal injection of urethane, which may cause an increase in plasma osmolality, and thus could lead to the secretion of vasopressin, as well as possibly affecting the baseline level of RSNA [30]. Such effects may therefore have affected the magnitude of the changes in RSNA evoked by microinjection of Ang II, but this would not explain the very marked differences in the magnitude of the sympathoinhibitory and depressor responses evoked from different subregions in the NTS.

Previous studies have demonstrated that there is a very high level of Ang II receptors, primarily of the AT1 subtype, in both the medial and commissural NTS [1,10,12,23,27]. These receptors are located on both nerve fibers and neuronal cell bodies $[5,9,11,14,28]$. There is some evidence that the AT1 receptor density varies according to the rostrocaudal level within the NTS. In particular, Healy and Zhang [10] found that the density of Ang II receptor binding in the medial and commissural NTS at the level $13.8 \mathrm{~mm}$ caudal to bregma (according to the atlas of Paxinos and Watson [19]) was approximately $60 \%$ higher than at levels $0.5 \mathrm{~mm}$ more rostral or caudal. The level $13.8 \mathrm{~mm}$ caudal to bregma is just caudal to the level of the obex [19], and thus corresponds to the rostrocaudal level of the NTS at which in the present study Ang II microinjections evoked the largest depressor and sympathoinhibitory responses.

Microinjection of glutamate into the medial NTS decreases arterial pressure and HR (e.g. [29]). Precise mapping studies, in which picomolar amounts of glutamate were injected into sites throughout the rostrocaudal extent of the medial and commissural NTS in the rat, show that marked depressor responses are evoked from sites in the NTS extending from the level immediately caudal to the obex to the level $1.5 \mathrm{~mm}$ rostral to the obex $[15,17]$. Thus, medial NTS neurons that are activated by Ang II and which evoke a depressor and sympathoinhibitory response appear to be a highly localized subset of the population of NTS depressor neurons.

Previous anatomical studies have shown that aortic nerve afferents in the rat, which are thought to arise exclusively from baroreceptors [25], have dense terminal fields in the dorsomedial NTS near the level of the obex [2,3], which corresponds closely to the region in which Ang II evoked 
large sympathoinhibitory responses. At the same time, aortic baroreceptor afferents also have dense terminal fields in other NTS regions, including the commissural NTS region caudal to area postrema [3], a region in which Ang II microinjections had little effect on RSNA or MAP. Thus, although it is possible that the sympathoinhibitory effect of Ang II is due to excitation of NTS neurons that are part of the baroreceptor reflex pathway, such neurons are likely to be only a subset of NTS neurons activated by baroreceptor inputs.

A recent study in the rat identified glutamatergic neurons within the NTS that are activated by baroreceptor inputs and which also project to the caudal ventrolateral medulla [31], and thus have properties indicative of interneurons within the baroreceptor reflex pathway $[4,8]$. The location of these neurons corresponds closely to the terminal fields of baroreceptor primary afferent fibers as described above [31], and includes the localized sympathoinhibitory region in the medial NTS just caudal to the obex. It is therefore possible that the Ang II-sensitive sympathoinhibitory neurons within this region are a subset of the barosensitive neurons in the NTS that project to and excite neurons in the caudal ventrolateral medulla that in turn project to and inhibit sympathetic premotor neurons in the rostral ventrolateral medulla.

Apart from the caudal ventrolateral medulla, there are also sympathoinhibitory neurons within the caudal midline medulla [20]. There is a projection from the NTS to the caudal midline medullary sympathoinhibitory region, but this arises mainly from the ventrolateral and dorsal parasolitary NTS subregions, with only a sparse projection from the medial NTS subregion at the level of the obex or more caudal to it [21]. Thus, while it is possible that the sympathoinhibitory effect of Ang II could be mediated in part via a projection from the NTS to the caudal midline medulla, the available anatomical evidence suggests that it is more likely to be mediated via a projection to the caudal ventrolateral medulla, as discussed above.

In the rat working heart-brainstem preparation, Paton and Kasparov [18] showed that Ang II microinjection into the NTS, in similar doses to those used in the present study, depresses the baroreceptor reflex control of heart rate, but in contrast to our results has little effect on baseline heart rate or perfusion pressure. In their study, however, microinjections were made into sites that were generally more caudal than the sympathoinhibitory region identified in our study, up to $0.5 \mathrm{~mm}$ caudal to the calamus scriptorius (i.e. approximately $1.1 \mathrm{~mm}$ caudal to the obex). Thus, the population of NTS neurons that subserve the Ang IImediated suppression of baroreceptor reflex bradycardia as observed in the study by Paton and Kasparov [18] are likely to be quite separate to those that mediate the renal sympathoinhibition described in the present study.

Although the sympathoinhibitory responses were usually accompanied by decreases in heart rate, in many cases the heart rate changed little or even increased. In addition, even when the heart rate did decrease, the time course of the bradycardia was often different to that of the sympathoinhibition. Furthermore, when a very low dose of $50 \mathrm{fmol}$ of Ang II was injected, there was no change in heart rate although a significant sympathoinhibition was still evoked. Thus, the results indicate that the sympathoinhibitory and bradycardic effects of Ang II in the medial NTS are mediated by separate mechanisms.

As mentioned in the Introduction, the depressor actions of Ang II in the NTS are mediated by AT1 receptors [7,13]. In normotensive rats, microinjection of the potent and highly selective AT1 receptor antagonist valsartan into the NTS has no effect on arterial pressure in normotensive rats, but does cause an increase in arterial pressure in spontaneously hypertensive rats (SHR)[13]. This indicates that the AT1 receptors that mediate sympathoinhibition are not tonically activated in normotensive rats, but are tonically activated by endogenous Ang II in SHR, possible as part of a compensatory response to the hypertension [13]. Thus, the Ang II-sensitive neurons within the medial NTS subregion could contribute to the long-term regulation of RSNA and arterial pressure, at least in hypertension.

In conclusion, this study has demonstrated that activation of AT1 receptors within a highly circumscribed region within the NTS can evoke marked renal sympathoinhibition and hypotension. The NTS neurons mediating the Ang IIevoked renal sympathoinhibition appear to be a subset of the population of depressor neurons within the nucleus. The available evidence also indicates that the AT1 receptors mediating renal sympathoinhibition are separate to those that modulate the baroreceptor reflex, but the circumstances in which these receptors are normally activated by endogenous Ang II, and their precise function in cardiovascular regulation, remain to be determined.

\section{Acknowledgments}

The study was supported by the National Health and Medical Research Council of Australia and the National Heart Foundation of Australia.

\section{References}

[1] C.O. Andrews, J.W. Crim, D.K. Hartle, Angiotensin II binding in area postrema and nucleus tractus solitarius of SHR and WKY rats, Brain Res. Bull. 32 (1993) 419-424.

[2] J. Ciriello, Brainstem projections of aortic baroreceptor afferent fibers in the rat, Neurosci. Lett. 36 (1983) 37-42.

[3] J. Ciriello, S.L. Hochstenbach, S. Roder, Central projections of baroreceptor and chemoreceptor afferent fibers in the rat, in: I.R.A. Barraco (Ed.), Nucleus of the Solitary Tract, CRC Press, Boca Raton, 1994, pp. $35-50$.

[4] R.A.L. Dampney, Functional organization of central pathways regulating the cardiovascular system, Physiol. Rev. 74 (1994) $323-364$.

[5] D.I. Diz, K.L. Barnes, C.M. Ferrario, Hypotensive actions of 
microinjections of angiotensin II into the dorsal motor nucleus of the vagus, J. Hypertens. 2 (1984) S53-S56.

[6] D.I. Diz, J.A. Jessup, B.M. Westwood, S.M. Bosch, S. Vinsant, P.E. Gallagher, D.B. Averill, Angiotensin peptides as neurotransmitters/ neuromodulators in the dorsomedial medulla, Clin. Exp. Pharmacol. Physiol. 29 (2002) 473-482.

[7] J.E. Fow, D.B. Averill, K.L. Barnes, Mechanisms of angiotensininduced hypotension and bradycardia in the medial solitary tract nucleus, Am. J. Physiol. 267 (1994) H259-H266.

[8] P.G. Guyenet, Role of the ventral medulla oblongata in blood pressure regulation, in: K.M. Spyer (Ed.), Central Regulation of Autonomic Functions, Oxford Univ. Press, New York, 1990, pp. 145-167.

[9] D.P. Healy, R. Rettig, T. Nguyen, T.M.P. Printz, Quantitative autoradiography of angiotensin II receptors in the rat solitary-vagal area: effects of nodose ganglionectomy or sinoaortic denervation, Brain Res. 484 (1989) 1-12.

[10] D.P. Healy, N. Zhang, Angiotensin II receptors in the solitary-vagal area of hypertensive rats, Hypertension 19 (1992) 355-361.

[11] J. Huang, Y. Hara, J. Anrather, R.C. Speth, C. Iadecola, V.M. Pickel, Angiotensin II subtype 1A (AT1A) receptors in the rat sensory vagal complex: subcellular localization and association with endogenous angiotensin, Neuroscience 122 (2003) 21-36.

[12] B.H. Hwang, J.W. Harding, D.K. Liu, L.S. Hibbard, C.M. Wieczorek, C.M.J.Y. Wu, Quantitative autoradiography of 125I-[Sar1, Ile8]angiotensin II binding in the brain of spontaneously hypertensive rats, Brain Res. Bull. 16 (1986) 75-82.

[13] N. Katsunuma, K. Tsukamoto, S. Ito, K. Kanmatsuse, Enhanced angiotensin-mediated responses in the nucleus tractus solitarii of spontaneously hypertensive rats, Brain Res. Bull. 60 (2003) 209-214.

[14] S.J. Lewis, A.M. Allen, A.J. Verberne, R. Figdor, B. Jarrott, F.A.O. Mendelsohn, Angiotensin II receptor binding in the rat nucleus tractus solitarii is reduced after unilateral nodose ganglionectomy or vagotomy, Eur. J. Pharmacol. 125 (1986) 305-307.

[15] V. Marchenko, H.N. Sapru, Different patterns of respiratory and cardiovascular responses elicited by chemical stimulation of dorsal medulla in the rat, Brain Res. 857 (2000) 99-109.

[16] R. Mosqueda-Garcia, C.J. Tseng, M. Appalsamy, D. Robertson, Cardiovascular effects of microinjection of angiotensin II in the brainstem of renal hypertensive rats, J. Pharmacol. Exp. Ther. 255 (1990) 374-381.

[17] D.O. Nelson, H.L. Cohen, J.L. Feldman, D.R. McCrimmon, Cardiovascular function is altered by picomole injections of glutamate into rat medulla, J. Neurosci. 8 (1988) 1684-1693.

[18] J.F.R. Paton, S. Kasparov, Differential effects of angiotensin II on cardiorespiratory reflexes mediated by nucleus tractus solitarii-a microinjection study in the rat, J. Physiol. 521 (1999) $213-225$

[19] G. Paxinos, C. Watson, The Rat Brain in Sterotaxic Coordinates, fourth ed., Academic Press, New York, 1998.

[20] J.R. Potas, R.A.L. Dampney, Sympathoinhibitory pathway from caudal midline medulla to RVLM is independent of baroreceptor reflex pathway, Am. J. Physiol. 284 (2003) R1071-R1078.

[21] J.R. Potas, K.A. Keay, L.A. Henderson, R. Bandler, Somatic and visceral afferents to the 'vasodepressor region' of the caudal midline medulla in the rat, Eur. J. Neurosci. 17 (2003) 1135-1149.

[22] R. Rettig, D.P. Healy, M.P. Printz, Cardiovascular effects of microinjections of angiotensin II into the nucleus tractus solitarii, Brain Res. 364 (1986) 233-240.

[23] B.P. Rowe, D.L. Saylor, R.C. Speth, Analysis of angiotensin II receptor subtypes in individual rat brain nuclei, Neuroendocrinology 55 (1992) 563-573.

[24] C.T. Sangaleti, A. Crescenzi, L.C. Michelini, Endogenous angiotensin and pressure modulate brain angiotensinogen and AT1A mRNA expression, Hypertension 43 (2004) 317-323.

[25] H.N. Sapru, E. Gonzalez, A.J. Krieger, Aortic nerve stimulation in the rat: cardiovascular and respiratory responses, Brain Res. Bull. 6 (1981) 393-398.

[26] J.P. Shaffer, Modified sequentially rejective multiple test procedures, J. Am. Stat. Assoc. 91 (1986) 826-831.

[27] K. Song, A.M. Allen, G. Paxinos, F.A.O. Mendelsohn, Mapping of angiotensin II receptor subtype heterogeneity in rat brain, J. Comp. Neurol. 316 (1992) 467-484.

[28] E.M. Szigethy, K.L. Barnes, D.I. Diz, Light microscopic localization of angiotensin II binding sites in canine medulla using high resolution autoradiography, Brain Res. Bull. 29 (1992) 813-819.

[29] W.T. Talman, M.H. Perrone, D.J. Reis, Evidence for L-glutamate as the neurotransmitter of baroreceptor afferent nerve fibers, Science 209 (1980) 813-815.

[30] G.M. Toney, Q.H. Chen, M.J. Cato, S.D. Stocker, Central osmotic regulation of sympathetic nerve activity, Acta Physiol. Scand. 177 (2003) 43-55.

[31] M. Weston, H. Wang, R.L. Stornetta, C.P. Sevigny, P.G. Guyenet, Fos expression by glutamatergic neurons of the solitary tract nucleus after phenylephrine-induced hypertension in rats, J. Comp. Neurol. 460 (2003) 525-541.

[32] J.W. Wright, W.S. Quirk, J.M. Hanesworth, J.W. Harding, Influence of aminopeptidase inhibitors on brain angiotensin metabolism and drinking in rats, Brain Res. 441 (1988) 215-220. 